\title{
La politique étrangère de la Belgique face à la France lors de la crise de la chaise vide (1965-1966). Rôle d'un «petit pays», poids d'une relation bilatérale
}

\author{
Vincent GENIN
}

L'Europe des Traités de Rome de 1957, les Six, ne compte pas moins de trois pays dits «petits»: les Pays-Bas, la Belgique et le Luxembourg. Ce concept de «petits pays», du moins dans les années 1960, occupe une place toute particulière dans l'imaginaire des trois «grands» (France, Italie, RFA), et à plus forte raison dans la France du général Charles de Gaulle (1958-1969), qui a été un acteur de premier plan de la construction européenne et, parfois, de l'opposition à celle-ci sous la forme de l'intégration (celle du Belge Paul-Henri Spaak et du Néerlandais Joseph Luns), lui préférant la voie confédéraliste de l'Europe des patries. ${ }^{1}$ Il convient toutefois de nuancer l'opposition souvent démontrée entre de Gaulle et Spaak, sur la question européenne, et même sur des dossiers multilatéraux en général. Bien que la France ait inquiété Bruxelles au printemps 1960, lors de l'éphémère "coexistence pacifique» franco-soviétique, à l'heure où l'on craint une déferlante communiste sur le Congo prochainement indépendant, Français et Belges, sur le dossier européen, ne cultivent aucun différend majeur avant l'été 1961. Au contraire, Spaak, ministre des Affaires étrangères depuis avril 1961, n'avait-il pas, en mai, lors de la visite du roi Baudouin à Paris, été considéré par le Quai d'Orsay comme un intermédiaire efficace destiné à convaincre Luns du bienfondé du confédéralisme? ${ }^{2}$

Toutefois, dès l'été 1961, Spaak se rallie à son homologue de La Haye, afin de prôner un «préalable anglais» à toute Union politique européenne. ${ }^{3}$ La France s'y oppose. Dès lors, une succession de lézardes vont poindre, certes rythmées de rémissions et de rechutes. L'échec du Plan Fouchet en 1962 et le veto français à toute adhésion britannique au Marché Commun, en janvier 1963, sur fond du traité francoallemand de l'Élysée (22 janvier 1963), que Bruxelles et d'autres «petits pays» considèrent comme un possible «axe Paris-Bonn» tout-puissant au sein des Six, ne contri-

1. Sur les questions traitées dans cette contribution, au sujet de Luns et de ses liens avec la France: G. MONGIN, Les rapports entre Joseph Luns et le général de Gaulle dans le cadre de la construction européenne de 1961 à 1965, mémoire de maîtrise en histoire, Paris I, 1996-1997; sur le concept de «petits pays»: M. DUMOULIN, G. DUCHENNE, A. VAN LAER (dir), La Belgique, les petits États et la construction européenne, Peter Lang, Bruxelles, 2003.

2. AMAEF-LC [Archives du ministère des Affaires étrangères de France-La Courneuve], Europe-Belgique (1961-1970), n¹36, série 11, sous-série 3, dossier 11), Bousquet à Laloy, 10.04.1961; Voir nos travaux : V. GENIN, L'ambassade de Belgique à Paris à l'époque de Marcel-Henri Jaspar (1959-1966). Activités, réseaux et opinions, vol.1, mémoire de master en histoire, ULg, 2011/12, pp.127-150; La visite du roi Baudouin au général de Gaulle en mai 1961: un climax dans les relations franco-belges, in: Museum Dynasticum, 1(2013), pp. 17-32.

3. J.-W. BROUWER, Les Pays-Bas et la querelle Spaak-de Gaulle, in: G. DUCHENNE, V. DUJARDIN, M. DUMOULIN (dir.), Paul-Henri Spaak et la France, Peter Lang, Bruxelles, 2007, pp.231-233. 
buent pas à atténuer ce climat de tension. ${ }^{4}$ Bien que des tentatives de relance auront lieu en 1963 et 1964, souvent à l'initiative de Paul-Henri Spaak, auquel, paradoxalement, la presse gaulliste (La Nation surtout) attribue de remarquables qualités, l'Europe en est réduite au statu quo ante. ${ }^{5}$ En 1965, la situation a mûri, et aux questions européennes, d'autres s'agrègent avec acuité. Elles sont d'ordre multilatéral, notamment la menace française de quitter les États-majors intégrés de l'OTAN, stationnés près de Paris. Or, cette injonction n'est pas nouvelle, puisqu'elle avait été au centre du mémorandum gaullien de 1958, suggérant, par ailleurs, de remettre en question le monopole atomique américain. Alors, Spaak, secrétaire-général de l'OTAN (1957-1961) s'était dit «stupéfait». ${ }^{6}$ Sept ans plus tard, la Belgique sera confrontée à une situation partiellement semblable.

La crise dite de la chaise vide, s'échelonnant de juin 1965 à janvier 1966, est sans doute celle au cours de laquelle la France a le mieux démontré sa capacité à obstruer la construction européenne, de manière isolée. La bibliographie sur le sujet est imposante. ${ }^{7}$ Nous allons y revenir. Cette crise montre surtout à quel point la politique étrangère de Paris est celle du général de Gaulle, celle du «Prince» pour paraphraser la terminologie réaliste du processus décisionnel, selon laquelle le chef d'État tient les rênes du pouvoir, au contraire de l'acception behavioraliste. ${ }^{8}$ La Constitution de 1958 implique une sensible évolution de la nature de ce processus décisionnel. ${ }^{9} \mathrm{Le}$ président, selon l'article 5 (al.2) est «garant du respect des accords de Communauté et des traités». C'est donc un domaine réservé. La primauté du président est, de plus,

4. V. GENIN, La Belgique face au traité de l'Élysée (juillet 1962-juillet 1963). Vers un axe Paris-Bonn ou une influence de Bruxelles?, in: Revue d'Allemagne et des pays de langue allemande, 1(2013), pp.183-198; Y. STELANDRE, Les pays du Benelux, l'Europe politique et les négociations Fouchet (26 juin 1959-17 avril 1962), in: Revue d'histoire de l'Intégration Européenne, 2(1996), pp.21-38.

5. L. WARLOUZET, Relancer la CEE avant la chaise vide. Néo-fonctionnalistes vs. fédéralistes au sein de la Commission européenne, in: Revue d'histoire de l'Intégration Européenne, 1(2008), pp. 69-86.

6. M. VAÏSSE, Aux origines du mémorandum de septembre 1958, in: Relations internationales, 2(1989), pp.256-257.

7. Plus généralement, nous renvoyons aux états de la recherche sur la construction européenne et la Belgique: M. DUMOULIN, L'historiographie de la construction européenne en Belgique, in: M. DUMOULIN, G. TRAUSCH (dir.), Historiographie des constructions européennes, Lettre d'Information des Historiens de l'Europe Contemporaine, 1-2(1992), pp.5-16; M. DUMOULIN, Y. STELANDRE, N. TOUSIGNANT, De impact van de Koude Oorlog op de Europese politiek van Belgïe, 1947-1962, in: M. VAN DEN WIJNGAERT, L. BEULLENS (dir.), Oost-West, West Best. Belgïe onder de Koude Oorlog, 1947-1989, Lannoo, Tielt, 1997, pp.92-103 et 263-266; M. DUMOULIN, La Belgique et la construction européenne: un essai de bilan historiographique, in: M. DUMOULIN, G. DUCHENNE, A. VAN LAER (dir), op.cit., pp.15-37.

8. D. BATTISTELLA, Théories des relations internationales, Presses de Science-Po, Paris, 2006, pp. 321-357; R. FRANK, Histoire et théories des relations internationales, in: R. FRANK (dir.), Pour l'histoire des relations internationales, PUF, Paris, 2012, pp.41-82.

9. Le concept de «processus décisionnel» est développé d'une manière formalisée par J.-B. DUROSELLE, La décision de politique étrangère. Esquisse d'un modèle-type, in: Relations internationales, 1(1974), pp.5-26. Voir aussi S. COHEN, Décision, pouvoir et rationalité dans l'analyse de la politique étrangère, in: M.-C. SMOUTS (dir.), Les nouvelles relations internationales. Pratiques et théories, Presses de la FNSP, Paris, 1998, pp.75-102. 
accrue par des moyens de communications plus rapides (le télex) réduisant la marge de manœuvre du diplomate, qui s'avère de moins en moins «plénipotentiaire». ${ }^{10}$

Les travaux récents de Philip Robert Bajon ont par ailleurs démontré à quel point la politiqueétrangèredelaFrancedurantlacrisedelachaisevide,dontilestunspécialiste,ne représentaitpasunblocmonolithique. ${ }^{11}$ SileQuaid'Orsay,leministèredesFinancesetle secrétariat général du Comité interministériel acceptent de débuter les discussions sur base des propositions de la Commission, le général de Gaulle ne l'entend guère de cette manière. Les premiers, qui auraient sans conteste été disposés à renforcer l'Assemblée parlementairemoyennantquelquecontrepartieàlaFrance,notammentsurl'instauration derèglesfinancières,n'ontpaspuconvaincrelechefdel'Étatencesens.Larupturedejuin 1965 entre Paris et le Marché Commun en cache une autre, celle, désormais apparente, entre le général de Gaulle et une partie non-négligeable de son administration. Dans le cadre denotre travail, ils'avèrera particulièrementutile de vérifier si cette dichotomie de la politique française était perçue par les autorités belges, ou si, au contraire, Bruxelles envisageait Paris comme un «monolithe».

Dans cette contribution, nous nous en tiendrons à étudier la manière dont la politique étrangère de la Belgique, souvent décrite comme unhonest broker, a tenté d'influeret de peser sur l'issue de cette crise de la chaise vide, pendant près d'un semestre. ${ }^{12}$

10. G. DEVIN, Sociologie des relations internationales, La Découverte, 2002, Paris, pp.50-52; G.-H. SOUTOU, La mécanisation du Chiffre au Quai d'Orsay ou les aléas d'un système technique (1948-1958), in: M. MERGER, D. BARJOT (dir.), Les entreprises et leurs réseaux: hommes, capitaux, techniques et pouvoirs, XIXe-XXe siècles, PUPS, Paris, 1998, pp.697-710.

11. Surl'attitudedelaFrancedurantlacrisedelachaisevide, ilconvientdeconsulterP.R.BAJON,TheEmpty ChairCrisisof 1965-1966.PerspectivesandInterestsoftheFrench Government, in:M.RASMUSSEN, A.-C.L. KNUDSEN (dir.), The Road to a United Europe. Interpretation of the Process of European Integration,PeterLang,Bruxelles,2009,pp.205-222; dumême:TheEuropeanCommissionandFrance in the Empty Chair Crisis of 1965-1966, in: J. ELVERT, S. SCHIRMANN (dir.), Zeiten im Wandel: Deutschland im Europa des 20. Jh. Kontinuität, Entwickelungen und Brüche, Peter Lang, Bruxelles, 2008,pp.159-176;DieKrisedesleerenStuhls 1965/66. Ursachen, VerlaufundFolgen, in:M.GEHLER (dir.), Vom Gemeinsamen Markt zur europaïschen Unionsbildung. 50 Jahre Römische Verträge 1957-2007,Böhlau,Vienne,pp.371-392.Cetauteuraégalementdéfenduunethèse(ParisIV-Universität Duisburg-Essen), en 2010: The Constitutionnal Crisis of European Communities 1965-1966. Origins, Development, Consequences. Bajonn'hésitepasàparlerdel'«aventurisme»dedeGaulle. VoiraussiW. LOTH, De Gaulle und Europa. Eine Revision, in: Historische Zeitschrift, 253(1991), pp.650-653.

12. Sur la Belgique et la crise: É. DESCHAMPS, Pacta sund servanda. Spaak, la France et la crise de la chaise vide (1965-1966), in: G. DUCHENNE, V. DUJARDIN, M. DUMOULIN (dir.), Paul-Henri Spaak et la France ..., op.cit., pp.200-224; É. DESCHAMPS, More than 'honest brokers'? Belgium, Luxembourg and the empty chair crisis (1965-1966), in: J.-M. PALAYRET, H.S. WALLACE, P. WINAND (dir.), Visions, votes and vetoes. The empty chair crisis and the Luxembourg compromise forty years on, Peter Lang, Bruxelles, 2006, pp.153-180. Sur la crise, de manière générale: J. NEWHOUSE, Collision in Brussels. The Common Market Crisis of 30 June 1965, New-York, Norton, 1967; N.P.LUDLOW, The European Community and the Crises of the 1960s. Negotiating the Gaullist challenge, Routledge, Londres, 2006; N.P. LUDLOW, Challenging French Leadership in Europe: Germany, Italy, the Netherlands and the Outbreak of the Empty Chair Crisis of 1965-1966, in: Contemporary European History, 8(1999), pp.231-248. En outre: M. VAÏSSE, La politique européenne de la France en 1965: pourquoi la chaise vide?, in: W. LOTH (dir.), Crises and compromises. The European project (1963-1969), Nomos, Baden Baden, 2001, pp.193-214; du même: La puissance ou l'influence?: la France dans le monde depuis 1958, Fayard, Paris, 2009, pp.108-110. 
Il ne s'agit donc pas d'une étude d'opinion. ${ }^{13}$ La notion d'honest broker avait été mise en avant, de manière convaincante, par Étienne Deschamps, dans un article axé sur l'attitude du Benelux (en particulier la Belgique et le Luxembourg) dans la crise de la chaise vide. Or, ici, notre ambition est de mieux comprendre l'impact de la relation bilatérale franco-belge sur cette crise, certes sans omettre un contexte plus large, sur base de documents jusqu'ici inexploités (en particulier le fonds Marcel-Henri Jaspar, ambassadeur de Belgique à Paris) et d'une littérature qui, depuis 2006, date de la publication du travail de Deschamps, s'est sensiblement étoffée. ${ }^{14}$ Il s'avère que, à plus d'une reprise, la Belgique a été un maillon essentiel dans le maintien du contact entre Paris et les Cinq, et fut souvent à l'origine de propositions, tantôt soutenues par les deux autres pays du Benelux, tantôt rejetées par ceux-ci. Cette configuration paraît donc propice à une étude plus particulière de la singularité de l'attitude belge, dans son unité, mais aussi sa diversité. Il sera surtout question de dégager les tenants et aboutissants du dispositifdiplomatique belge à l'égard de la France, principal acteur de la situation, afin de sortir de l'ornière que représente cette crise. Ce concept de crise est, traditionnellement, d'autant frappé au coin de l'incertitude qu'il est, par principe, difficile d'en définir le début et la fin. Or, chacun semble s'accorder sur le fait que celle-ci est clairement circonscrite, entre juin 1965 et janvier 1966. Enfin, à l'heure où le multilatéralisme (OTAN, ONU, UEO, Marché Commun, tripartites) semble avoir définitivement éclipsé le bilatéralisme, le cas de figure que nous présentons se situe à un niveau bilatéral (Paris-Bruxelles), et tente de dégager le poids relatif de ce dernier, dans un contexte multilatéralisé. ${ }^{15}$

Plusieurs sources d'archives ont été mobilisées pour répondre à ces questions. Retenons les principales. Citons en premier lieu celles du ministère des Affaires étrangères de Belgique (correspondance de l'ambassade à Paris), et celles de MarcelHenri Jaspar, ambassadeur de Belgique à Paris de 1959 à 1966, déposées aux Archives Générales du Royaume (I 226). Les quelques lacunes des collections du ministère sont, en général, opportunément comblées par le Fonds Jaspar, contenant l'ensemble des rapports qu'il a rédigés durant sa carrière. Sa correspondance privée a également été consultée de manière exhaustive. Par ailleurs, les documents de la Fondation PaulHenri Spaak et ceux du Centre d'Étude de l'Europe Contemporaine de Louvain-laNeuve (fonds Paul Van Zeeland) ont contribué à offrir d'autres angles d'approches.

13. Cf. L. LEBRUN, La crise de la "chaise vide": analyse de deux quotidiens belges francophones, mémoire de master, UCL, 2010-2011.

14. É. DESCHAMPS, More than 'honest brokers'? ..., op.cit.

15. T. DE MONTBRIAL, L'action et le système du monde, PUF, Paris, 2011, pp.110-113; J.D. SINGER, The Level-of-Analysis Problem in International Relations, in: H.R. NAU (eds), International Relations in Perspective, CQPress, Washington, 2010, pp.84-97; M. VAÏSSE, Une invention du XIXème siècle, in: B. BADIE, G. DEVIN (dir.), Le multilatéralisme. Nouvelles formes de l'action internationale, La Découverte, Paris, 2007, pp.13-22; A. PLANTEY, Négociation diplomatique et arbitrage commercial international, in: B. BADIE, A. PELLET (dir.), Les relations internationales à l'épreuve de la science politique. Mélanges Marcel Merle, Economica, Paris, 1993, pp.371-381. 


\section{Rupture douce et silences de Paris}

La principale source d'information et le principal levier d'action de la politique étrangère belge à l'égard des affaires françaises est l'ambassade de Belgique à Paris. Jusqu'à la crise de la chaise vide, cette institution a souvent été reléguée au second plan de l'échiquier diplomatique belge: éviction de l'ambassadeur durant la crise congolaise, à l'été 1960, et rôle de simple observateur des remous du Marché Commun, en particulier depuis le printemps 1961, date de l'arrivée aux affaires du ministre des Affaires étrangères Paul-Henri Spaak, tenant du «préalable anglais» à toute Union politique. Européen convaincu, mais souvent décrit comme un opposant historique de Spaak, l'ambassadeur à Paris, Marcel-Henri Jaspar, est lié à des européistes de premier ou de second plan, à l'instar de l'ancien Premier ministre Paul Van Zeeland, ou de Richard Coudenhove-Kalergi. ${ }^{16}$ Fondateur de Paneuropa et du Mouvement Européen, ce dernier, pour avoir donné en 1962 son blanc-seing aux thèses européennes de de Gaulle, a été marginalisé par de nombreux européistes. ${ }^{17}$ Réticent à un rapprochement franco-allemand trop poussé, puis inquiet devant une distanciation excessive entre Paris et Bonn (abandon possible d'une RFA réarmée à l'influence soviétique), Marcel-Henri Jaspar a été un observateur attentif de la politique européenne. Il n'y a toutefois jamais été impliqué en tant qu'acteur, jusqu'à l'été 1965.

Il est le témoin des prémisses de la crise de la chaise vide. Mais qu'elle en est l'origine, en quelques mots? La prévision d'instaurer, dès le 1er janvier 1966, au sein des Conseils européens, le vote majoritaire en lieu et place du vote à l'unanimité, les difficultés posées par la France dans le financement de la Politique Agricole Commune (PAC) et la volonté réitérée par Spaak de créer une commission unique, cristallisent les divergences. ${ }^{18}$ Celles-ci ont été accentuées en juin 1965. Le 4 juin, Marcel-Henri Jaspar relate à Spaak la réunion des ministres de la Défense de l'OTAN, qui s'est tenue à Paris. La substance lui en a été communiquée par Charles Lucet, le directeur des Affaires Politiques (DAP) du Quai d'Orsay. Pierre Messmer, ministre des Armées, lisant une note préparée dans la précipitation, avait, dans un premier temps, déclaré que la France ne sortirait pas de l'OTAN avant décembre, c'est-à-dire

16. AGR [Archives Générales du Royaume], FMHJ, n491, Jaspar à Coudenhove-Kalergi, 13.02.1965; Coudenhove-Kalergi à Jaspar, 9.3.1965; Coudenhove-Kalergi à Jaspar, 12.12.1965. CoudenhoveKalergi espérait trouver une oreille attentive à ses projets au Palais Royal, par l'intermédiaire de Jaspar. En vain. Voir aussi V. GENIN, Le plus gaullien des Belges? Marcel-Henri Jaspar face au général de Gaulle (1940-1966), in: Nouveaux regards sur de Gaulle, la période gaullienne et la Belgique (à paraître).

17. T. SALMON, W. NICOLL (dir.), Building European Union, Carnegie, Preston, 1997, p.7; M. DUMOULIN, V. DUJARDIN, Paul Van Zeeland, Bruxelles, Racine, 1997, pp.214-215; Chapitre IV de M. DUMOULIN, A.-M. DUTRIEUE (dir.), La Ligue européenne de coopération économique (1946-1981). Un groupe d'étude et de pression dans la construction européenne, Peter Lang, Bruxelles, 1993, pp.109 f.; C. LANNEAU, Du gaullisme à la droite radicale. Les étranges 'compagnons de route' belges de l'Europe gaullienne, in: O. DARD (dir.), Doctrinaires, vulgarisateurs et passeurs des droites radicales au XXème siècle (Europe-Amériques), Peter Lang, Bruxelles, 2012, pp.99-126.

18. La France tient à ce système de l'unanimité étant donné qu'il donne à son éventuel veto la capacité de rejeter toute proposition. Le vote majoritaire serait donc un échec pour de Gaulle. 
avant la réélection éventuelle du général de Gaulle. Quelques heures plus tard - suite à un «recadrage» du ministre des Affaires étrangères, Maurice Couve de Murville Messmer tempère ses propos et opère une volte-face. Le malaise s'installe dans la réunion. La tension est également perceptible du côté du MRP Maurice Schumann, président de la commission des Affaires étrangères de l'Assemblée nationale, dont les analyses étaient consultées avec attention par les agents belges. ${ }^{19}$ Ce trait est de plus renforcé par la proximité qui existe entre Spaak et Schumann, anciens alliés dans le combat pour la CED, en 1954. Schumann veut rassurer les Belges et publie un article «optimiste» dans la revue Entreprise. Mais trop optimiste selon Bruxelles. ${ }^{20}$

Sur le plan du Marché Commun, de Gaulle concède au chancelier allemand Ludwig Erhard une rencontre des Six en octobre. Le Quai d'Orsay n'y voit qu'une volonté de désamorcer artificiellement la situation et de soutenir, accessoirement, la reconduction du chancelier, que l'Élysée, qui n'a certes jamais entretenu d'aussi bonnes relations avec l'économiste et trop atlantiste Erhard qu'avec Adenauer, préfère toutefois à un SPD. ${ }^{21}$ Le 22 juin, dans sa déclaration de politique étrangère, le Premier ministre Georges Pompidou, au contraire d'une frange plus dure des gaullistes, n'envisage pas la sortie pure et simple de l'OTAN. Mais le principe de la Force multilatérale intégrée, tel qu'il se profile, est refusé net. ${ }^{22}$ Le 30 juin, les protagonistes du Conseil ne parviennent pas à boucler un accord financier concernant le financement de la PAC. Ce refus, et ce sujet, qui bloquent le traité de Rome depuis trois ans, signifient, in petto, que la France, malgré son ton rassurant, veut renégocier tout le traité de $1957 .{ }^{23}$ Le 1er juillet, malgré les tentatives de sauvetage de Spaak, Couve de Murville quitte le Conseil. ${ }^{24}$ Ce geste d'indépendance est tout à fait caractéristique de la phase dite «opératoire», pour reprendre la formule de Maurice Vaïsse, selon laquelle la France mène une politique que l'on qualifierait en Belgique, mutatis mutan-

19. V. GENIN, L'ambassade ..., op.cit., vol.2, p.283.

20. FPHS [Fondation Paul-Henri Spaak], F302/D5677, Jaspar à Spaak, 04.06.1965.

21. Ibid., Jaspar à Spaak, 4.6.1965; voir aussi B. SCHOENBORN, Les réticences du Chancelier Erhard à l'égard de la France (1963-1966), in: Relations Internationales, 2(2006), pp.3-13.

22. FPHS, F302/D5675, Jaspar à Spaak, 22.06.1965. J.F. Kennedy avait ambitionné, en juillet 1962, de doter les armes nucléaires européennes de la «double-clé» (aspect multilatéral), impliquant que celles-ci se trouvent sous la houlette de l'OTAN (aspect multinational). Suite aux Accords de Nassau de décembre 1962, la Grande-Bretagne adhère à ce système, à l'inverse de la France. Le Premier ministre britannique, Harold Macmillan, accepte ainsi que la défense britannique se dote de fusées américaines Polaris, que l'on pourrait munir de têtes nucléaires anglaises. Londres n'avait pas consulté Paris au préalable. Cette Multilateral Force durera encore près de deux ans (1965), tout comme l'ANF (Atlantic Nuclear Force). L'accord de Nassau avait par ailleurs été invoqué comme prétexte à la rupture du 14 janvier 1963, au cours de laquelle de Gaulle a opposé son veto à l'adhésion britannique au Marché Commun. Voir e.a. G. BOSSUAT, Faire l'Europe sans défaire la France. 60 ans de politique d'unité européenne des gouvernements et des présidents de la République française (1943-2003), Paris, Peter Lang, 2005, p.105; J. RAFLIK, L'Europe en quête de puissance. La France entre européisme et atlantisme depuis 1945, in: M. AFFINITO, G. MIGANI, C. WENKEL (dir.), Les deux Europes, Peter Lang, Bruxelles, 2009, p.274.

23. A. PEYREFITTE, C'était de Gaulle, Gallimard, Paris, 2000, p.894.

24. M. DUMOULIN, Spaak, Racine, Bruxelles, 1999, pp.659-660. 
dis, des «mains libres». ${ }^{25} \mathrm{C}$ 'est l'acte fondateur de la crise européenne dite de la chaise vide.

Le 2 juillet, le premier conseiller de Jaspar, Werner de Mérode, rencontre Étienne Burin des Roziers, secrétaire général à la présidence. Il n'y trouve aucune animosité à l'égard de Spaak, comme lors de ses tentatives de relance en $1964 .{ }^{26}$ Couve de Murville a même déclaré que «Spaak a été d'une parfaite correction à travers toute la négociation». ${ }^{27}$ Jeter l'anathème sur le Belge, il est vrai, aurait été malaisé à justifier. Dès ces premiers jours de crise, la Belgique dispose les premiers jalons de son action en vue d'une relance. Soufflant le chaud et le froid, le Quai d'Orsay suggère à Spaak de maintenir son cap et de «désembourber» la situation, une fois la crise ministérielle belge dissipée. En effet, depuis les élections de mai 1965, de longues négociations se tiennent afin de former un gouvernement capable de prendre la relève du cabinet Spaak-Lefèvre d'avril 1961. Or, le moment est passablement mauvais pour Spaak: ses voix de préférence ont chuté de 63.000 à 34.000 , les socialistes opèrent un sensible reflux au bénéfice des libéraux et des partis communautaires, et sa place au sein du Parti socialiste est de plus en plus hypothéquée par les jeunes cadres du parti. Il doit maintenir l'équilibre entre crise interne et crise européenne. Son statut à l'échelle internationale sera d'ailleurs sans doute un des éléments qui contribueront à le reconduire aux Affaires étrangères, dans le gouvernement formé par Pierre Harmel, le 27 juillet 1965 .

Dès la résorption de la crise ministérielle, il convient de ne se consacrer qu'à l'Europe et, à en croire les agents belges à Paris, attendre le mois de septembre pour agir en direction de Paris sur la question de la PAC. La rentrée est souvent l'occasion de mouvements sociaux, paysans et industriels. Ce fond de tensions sociales donnerait plus d'intensité à la politique belge, face à un de Gaulle tenu par l'échéance des élections présidentielles du 5 décembre. Bruxelles dresse donc un premier calendrier, celui d'une résolution de la crise, sous son impulsion, vers septembre-octobre. ${ }^{28}$

Mais ce calendrier n'est que théorique. La situation mûrira plus rapidement que prévu, et Bruxelles tentera la relance auprès de Paris avant ces échéances. Il apparaît d'abord aux Belges qu'ils bénéficient d'un allié: la presse française. La gaulliste Nation attaque les a priori supranationaux de Bonn et de La Haye, mais la Belgique, en revanche, y est remarquablement épargnée. ${ }^{29}$ L'ex-sous-directeur à l'Europe oc-

25. Ce thème est développé dans M. VAÏSSE, La Grandeur. Politique étrangère du général de Gaulle (1958-1969), Fayard, Paris, 1998.

26. V. GENIN, L'ambassade ..., op.cit., vol. 2, pp.305-308.

27. AMAEB [Archives du ministère des Affaires étrangères de Belgique], $\mathrm{n}^{\circ} 14642$,De Mérode à Spaak, 03.07.1965.

28. AMAEB, ${ }^{\circ} 14642$, De Mérode à Spaak, 03.07.1965.

29. Nous renvoyons à l'ensemble du travail de B. BRICHEUX, La politique européenne de la France vue par le ministère des Affaires étrangères de Belgique (1961-1966): apport d'un observateur privilégié et de la presse périodique, mémoire de maîtrise, UCL, 2009/10. Il note que cette appréciation émane des «avis parisiens», ce qui est assez vague. Le fait que La Nation, dont, depuis plusieurs années, certaines colonnes furent d'une grande sévérité pour Spaak, rédige ces lignes probelges, porte une signification particulière (AMAEB, n 14642, Jaspar à Spaak, 06.07.1965. 
cidentale du Quai d'Orsay (1959-1961), Jean François-Poncet, ancien critique de Spaak, n'a cessé de voir en lui le partisan du «préalable anglais». ${ }^{30}$ Or, c'est sous sa plume que Le Figaro loue sans ambages l'attitude du ministre belge. Mais, outre cet atout, la principale inconnue pour les Belges est l'attitude que compte adopter de Gaulle. Le Quai d'Orsay est particulièrement désinformé et n'a été mis au courant de la crise que le 6 juillet par l'Élysée. ${ }^{31}$ Il n'existe donc, en juillet 1965, aucune source capable de livrer aux Belges la pensée du général. Ce dernier compte, par ce moyen, accentuer la pression sur les Cinq. Pour sa part, dans le même esprit, Couve de Murville refuse de rencontrer, le 26 juillet, son homologue italien Amintore Fanfani. $^{32}$

\section{Rétablir un contact Paris-Bruxelles: une voie bilatérale?}

En août 1965, Marcel-Henri Jaspar est situé au premier plan du dispositif de Spaak. Il sera un intermédiaire entre son ministre et Couve de Murville et, si nécessaire, l'Élysée. Un petit dossier des Papiers Jaspar est particulièrement instructif à cet égard. L'ambassadeur a proposé d'écourter ses vacances. Lors d'une discussion avec de Mérode et Étienne Davignon, le premier collaborateur de Spaak, l'ambassadeur se propose de rencontrer Couve de Murville; ${ }^{33}$ Spaak souhaite qu'il s'entretienne plutôt avec de Gaulle. Le 10 août, Davignon estime que Jaspar doit obtenir ces entretiens avant la fin du mois d'août, afin de «confronter les renseignements recueillis avec ceux de nos partenaires». ${ }^{34}$ C'était sans compter sur une absence prolongée de Paris de Couve de Murville, jusqu'environ le 25 août. Cette disposition de calendrier permet ainsi à Jaspar de suivre l'affaire depuis Antibes, où il séjourne. La situation est dans une sérieuse ornière. Il confie ses craintes au secrétaire général des Affaires étrangères, son ami Jean Van den Bosch:

«Évidemment, il me faudra voir Couve avant la fin du mois. Je le déplore d'autant plus qu'à mon avis les jeux sont faits. La France acceptera-t-elle les nouvelles propositions de la Commission Hallstein? Peut-être! Mais elle mettra des conditions que les Cinq ne pourront accepter. Peut-être pourriez-vous tâter le terrain au Quai où vous avez d'heureux contacts? Le Gal [sic] cogite, sous les deux clochers et je doute qu'il soit possible de l'influencer». ${ }^{35}$

Manifestement, aucun contact solide n'a été obtenu par les Belges du côté du Quai d'Orsay. Aucune information ne perle. Couve de Murville ne souhaite fixer aucun entretien avant le 31 août. Davignon insiste pour que la date soit avancée; chaque

30. AMAEB, $n^{\circ} 14363$, Jaspar à Spaak, 15.09.1964.

31. AMAEB, ${ }^{\circ}$ 14642, Jaspar à Spaak, 06.07.1965; De Mérode à Spaak, 07.07.1965.

32. M. COUVE DE MURVILLE, Une politique étrangère (1958-1969), Plon, Paris, 1971, pp.332-337; AMAEB, n¹4642, Jaspar à Spaak, 23.07.1965.

33. V. DUJARDIN, Pierre Harmel. Biographie, Le Cri, Bruxelles, 2004, p.306).

34. AGR, FMHJ, n²608, Davignon à de Mérode, 10.08.1965.

35. Ibid., Jaspar à Van den Bosch, 16.08.1965. 
journée compte pour les Belges, tandis que les Français veulent surtout gagner du temps. Le 23, de Mérode adresse une longue lettre à Davignon. Elle ne peut satisfaire Spaak, mais, pire, certains mots du premier conseiller semblent désapprouver l'attitude de la Commission et de son président, Walter Hallstein. Par ailleurs, il diverge avec le cabinet de Spaak sur le calendrier des entretiens: une entrevue avec l'Élysée ne sera efficace que si elle se situe entre le 1er et le 3 septembre, c'est-à-dire une semaine avant la conférence de presse du général de Gaulle, prévue le $9 .{ }^{36}$ Au mutisme de l'Élysée et aux silences du Quai d'Orsay s'agrège ce différend belgo-belge. Les circonstances ne sont pas encore mûres pour agir.

Une première lucarne, livrant la position de la France, s'ouvre pourtant aux Belges sous une forme pour le moins informelle. En effet, il se trouve que Werner de Mérode passe plusieurs jours en Haute-Savoie chez Jean-Marc Boegner, son cousin par alliance, et, surtout, représentant permanent de la France auprès des Communautés européennes. ${ }^{37}$ Boegner est donc le visage de cette «chaise vide». Il était le neveu de Paul Bargeton, ambassadeur de France à Bruxelles de 1937 à 1940, connu de tout agent du Quai d'Orsay pour avoir interrogé les jeunes candidats, lors des examens diplomatiques. ${ }^{38}$ Voilà donc une coïncidence; encore faut-il l'exploiter. Après quatre jours de silence, la glace est brisée et Boegner en vient à aborder la question de la crise à de Mérode. Proche du général, il affirme que celui-ci veut poursuivre la coopération à Six, dans les domaines de l'harmonisation fiscale ou des transports. Boegner concède que ses petites phrases concernant le «volapük» et les «technocrates apatrides» n'ont pas détendu l'atmosphère, mais il estime que les positions de Walter Hallstein ont durci la position du président. L'élargissement des compétences de la commission, en dehors des limites du traité de 1957, ne pouvait que susciter son ire. Werner de Mérode paraît convaincu par son parent et collègue et envisage une action de la Belgique. Bien qu'il semble comprendre l'attitude française, comme précisé plus haut, il estime que le Royaume a une «carte» à jouer: proposer à Paris les propositions d'Hallstein revues à la baisse, «en association ou non avec d'autres partenaires». ${ }^{39} \mathrm{Ce}$ dernier point suggère l'éventualité d'une démarche bilatérale. L'agent belge ne se cantonne pas à une démarche belge en coopération avec ses partenaires, ce qui semblait être l'objectif du vicomte Davignon: la Belgique pourrait ipso facto tenter une manœuvre en cavalier seul.

Mais il ne serait pas pertinent de réduire la politique étrangère de la Belgique, cœur de notre propos, aux considérations d'un agent. Ses vues ne convainquent que partiellement l'ambassadeur à Paris. Ce dernier admet en effet que rien de décisif ne se décantera avant la conférence de presse du général de Gaulle, fixée le 9 septembre. Ce détail implique par ailleurs qu'il ne croit guère à la portée de sa prochaine visite

36. Ibid., D'Avernas à Jaspar, 19.08.1965; Davignon à d'Avernas, 20.08.1965; De Mérode à Davignon, 23.08.1965.

37. Assez curieusement, Boegner ne se souvient pas de cet épisode savoyard dans le témoignage qu'il livre en 1999 à Marie-Thérèse Bitsch. Il affirme qu'après son départ de Bruxelles, «je suis resté tranquillement chez moi» (G. BOSSUAT, op.cit., pp.417-419).

38. R. PEYREFITTE, Les Ambassades, Flammarion, Paris, 1951, pp.13-14.

39. AGR, FMHJ, n²608, De Mérode à Davignon, 23.08.1965. 
chez Couve de Murville. Jaspar propose que le 10, l'ambassadeur Van der Meulen rédige un mémorandum récapitulatif, sans privilégier une issue particulière. ${ }^{40}$ Mais, au contraire de son collaborateur, Jaspar ne songe pas à une démarche bilatérale Bruxelles-Paris. Bien que, comme lui, il estime que le responsable de cette crise soit la Commission européenne, il ne juge pas utile de la pointer du doigt trop ostensiblement. Il propose donc à Bruxelles de soumettre à Bonn, La Haye, Luxembourg et Rome, lors de la prochaine rencontre des Six, en octobre, une note mâtinée de cet esprit conciliateur et de préparer un projet de communiqué allant dans le même sens: prôner une construction européenne dans le cadre des traités de 1957, sans mentionner ou accuser la Commission. ${ }^{41}$ Enfin, côté belge, il est fortement déconseillé d'aborder, lors de cette réunion, la totalité des contentieux en suspens: fusion des exécutifs, compétence et mode d'élection de l'Assemblée parlementaire européenne, reconduction du mandat des commissaires etc. ${ }^{42}$ Ces questions seront traitées en leur temps. Il faut renouer avant tout et opérer une relance européenne.

Malgré l'opposition de l'ambassade belge à Paris, un entretien Jaspar-Couve de Murville aura bien lieu le 31 août. Le cabinet de Spaak y tenait particulièrement et tient à «briefer» Jaspar peu avant le rendez-vous. La diplomatie belge fondait beaucoup d'espoir en ce dernier; or, il fut relativement décevant. Au contraire des Belges, Couve de Murville souhaite que l'ensemble du contentieux soit abordé lors de la réunion des Six d'octobre, en plus du mémorandum agricole de la commission Hallstein. On s'accorde toutefois sur la nécessité de «recadrer» la marge de manœuvre de la Commission européenne, à l'intérieur des limites circonscrites en 1957. Le ministre rompt peut-être le silence du Quai d'Orsay mais il maintient la ligne maîtresse de ce dernier: gagner du temps. Bruxelles avait escompté faire pression sur Paris en misant sur l'échéance des présidentielles de décembre. Toutefois, il se trouve que celles-ci inspirent un sentiment inverse à Paris: toute grande négociation européenne sera compromise par la mobilisation de la classe politique française par ce scrutin. De plus, à Bonn, aucun gouvernement ne sera installé avant octobre. Il est promis aux Belges qu'un deuxième entretien bilatéral aura lieu le 10 septembre, au lendemain de la conférence de de Gaulle. ${ }^{43}$

Jaspar confie à Spaak que, malgré ces réserves, il existe une réelle identité de vues entre Bruxelles et Paris, entre Spaak et Couve de Murville: trop grande passivité de Fanfani face à la crise, hypothèques sur la volonté allemande de pousser plus loin le Marché Commun et réticence systématique des Pays-Bas à l'égard de la France, qui,

40. Spaak demandera à Van der Meulen, le 20 septembre, que l'on n'aborde pas ouvertement l'aspect politique de la crise au la COREPER. Celui-ci doit pouvoir se réunir sans la France, ce qui est un signe fort à destination de celle-ci. Mais, toute décision de poids est pour l'heure inconcevable en son absence (É. DESCHAMPS, Pacta ..., op.cit., p.205).

41. Aide-mémoire de Jaspar à Spaak, s.d. (entre le 23 et le 26 août 1965, selon nous).

42. O. COSTA, Le Parlement européen dans le système décisionnel de l'Union Européenne: la puissance au prix de l'illisibilité, in: Politique européenne, 2(2009), pp.129-155.

43. AGR, FMHJ, n²608, Aide-mémoire de Jaspar à Spaak, 31.08.1965. 
comme le précise Couve de Murville, en boutade, «en sont encore à craindre Louis $\mathrm{XIV} \gg .{ }^{44}$

Le rôle de la Belgique revêt à ce moment précis un intérêt non-négligeable. Elle est la seule que de Gaulle et son ministre des Affaires étrangères ne vouent pas aux gémonies. Le 8 septembre, de Gaulle réserve encore devant Alain Peyrefitte, ministre de l'Information, une critique acerbe à destination de Bonn, Rome et La Haye, en épargnant Bruxelles et Luxembourg. Mais le président français ne souhaite accorder aucune audience d'ici la fin de l'année, avortant ainsi les espérances des Belges. Il l'avait déjà décidé le 26 juillet. ${ }^{45}$ La conférence du 9 septembre n'apporte aucun élément novateur: rejet de la Commission, défense d'une relance de la CEE et négation d'une sortie de l'OTAN, à condition que la France ne soit pas contrainte à l'intégration. Le 15 septembre, le Directeur de l'Europe confirme aux agents belges que «le mot d'ordre sera immobilisme jusqu'en janvier». Toutefois, observons que, dès le lendemain, le directeur en question revient sur sa déclaration de la veille et affirme que des discussions entre gouvernements sont tout à fait envisageables, «en dehors de toute conjecture électorale». ${ }^{46} \mathrm{Il}$ a sans doute été rappelé à l'ordre par Couve de Murville, qui tient à maintenir le contact avec les autorités belges. Nous y voyons surtout un besoin pour le Quai d'Orsay de nager entre deux eaux: d'une part, battre froid les diplomates belges serait sacrifier inutilement un éventuel atout dans la dure négociation qui s'annonce en janvier, mais, d'autre part, de Gaulle semble bien décidé à ne pas bouger d'ici l'issue du scrutin.

\section{La tentative de relance de Spaak}

De son côté, Paul-Henri Spaak, dont le naturel entreprenant revient au galop, tente une relance, quitte à ce que la Commission européenne soit éludée. Cette attitude est par ailleurs privilégiée par Couve de Murville. Spaak fait donc un pas vers Paris. Afficher l'importance du Conseil des ministres des Affaires étrangères est par ailleurs primordial. Les réactions françaises ne se font pas attendre et précèdent même la parution d'un article du ministre, le 25 octobre. Un rapport du 8 octobre en fait état. Comme en 1964 et en juillet 1965, la presse française accueille ce projet avec enthousiasme, ou, au pire, avec scepticisme. Le Monde et Le Populaire du 25 septembre

44. On connaît le parallèle classique entre de Gaulle et Louis XIV. Déjà en janvier 1962, le sénateur social-chrétien Étienne de la Vallée-Poussin, dans Le Soir (20-21/1/1962), s'en était fait l'écho; il récidive en 1968 (V. DUJARDIN, Étienne de la Vallée-Poussin, in: Nouvelle Biographie Nationale, vol.9, Académie Royale de Belgique, Bruxelles, 2007, p.123).

45. «Alain Peyrefitte: Vous ne pensez pas que les entretiens bilatéraux avec Luns et Spaak etc. peuvent faire avancer les choses? - de Gaulle: Non. Ma résolution est absolument prise. Je suis décidé à ne fixer aucune entrevue d'ici la fin de l'année. Ceci dit entre nous: à la fin de l'année, on les ramassera à la petite cuillère» (A. PEYREFITTE, op.cit., p.890).

46. AMAEB, $\mathrm{n}^{\circ} 14642$, Jaspar à Spaak, 09.09, 16.09 et 17.09.1965. 
abondent dans le sens du Belge. ${ }^{47}$ Le Figaro du 25 septembre oppose Spaak à un autre européen, Jean-Charles Snoy, ancien secrétaire général du ministère des Affaires économiques, reconverti dans la Banque Lambert, quant au sort à réserver à la France. Snoy vient de publier son opinion dans la Revue Générale belge. Il affirme qu'une Europe sans la France n'était pas inconcevable. ${ }^{48}$

Comme le dit avec justesse Étienne Deschamps, Spaak manifeste également son impatience à l'égard de Paris, notamment à cause de la situation bloquée du budget des Communautés. ${ }^{49}$ Toutefois, il convient de préciser que Spaak considère l'expédient proposé par Snoy comme la solution «du désespoir». La presse française ne s'y trompe pas et tresse des lauriers à Spaak. Mais, ce bon accueil de la presse ne conditionne pas l'attitude du Quai d'Orsay. Après un entretien informel avec un représentant d'une chaîne de journaux allemands à Bruxelles et à Paris, Jaspar apprend que Couve de Murville aurait confié à Fanfani qu'il rejetait le projet de relance de Spaak. Au Quai, les fonctionnaires gagent que cet immobilisme durera jusqu'au moins la fin de l'année.

Cette attitude de Couve de Murville montre bien que, de toute façon, la France ne souhaitait aucune rencontre, étant donné que les détails du projet de Spaak ne seront dévoilés que le 25 octobre, c'est-à-dire après la déclaration prétendue du ministre français à Fanfani. Les agents belges étaient invités à la plus grande confidence au sujet de ce projet. ${ }^{50}$ Toutefois, quelques jours avant que ne soit publié ce plan, comme pour ménager les Belges, le directeur des Affaires économiques du Quai d'Orsay, Olivier Wormser, remet, en primeur, une note à de Mérode. Il s'agit d'une déclaration que le ministre français prononcera quelques heures plus tard, sur une idée de Geoffroy de Courcel, ambassadeur à Londres. Il y est question d'un rejet du vote majoritaire et d'une volonté d'aboutir à un accord politique. En somme, rien de décisif. Il y est surtout question d'un rejet français du plan de relance de Spaak. Or, cette note française date du 20 octobre, et semble connaître en détail le plan qui sera dévoilé cinq jours plus tard par le Belge. Est-ce le fruit de fuites? Sans nul doute. La presse ayant déjà éventé plusieurs indiscrétions. Bien qu'il s'agisse d'un «geste de courtoisie» à l'égard des Belges, seuls a en être informés à Paris, ce n'en est pas moins un refus de la relance. ${ }^{51}$

47. M.-T. BITSCH, Spaak et la France dans le miroir du journal Le Monde, in: G. DUCHENNE, V. DUJARDIN, M. DUMOULIN (dir.), op.cit., pp.266-268.

48. M. DUMOULIN, V. DUJARDIN, Jean-Charles Snoy, Le Cri, Bruxelles, 2011, pp.372-376; J.-C. SNOY et D'OPPUERS, Faire l'Europe sans la France?, in: Revue Générale belge, 9(1965), pp. 19-33. L'opinion de Snoy reçoit un accueil favorable dans plusieurs journaux, à l'instar du Soir, sous la plume de de la Vallée-Poussin (15.09.1965, pp.1-2) et du Monde, sous celle de Louis Duquesne Watelet de la Vinelle (23.09.1965, p.21).

49. E. DESCHAMPS, Belgium ..., op.cit., p.170.

50. AMAEB, n¹4642, Jaspar à Spaak, 08.10.1965.

51. Ibid., Jaspar à Spaak, 20.10.1965. Le plan Spaak avait été proposé par la Belgique unilatéralement. En effet, Spaak avait tenté d'obtenir l'appui des deux autres pays du Benelux, mais, pris par le temps, en l'absence d'une réponse rapide de leur part, et au regard des atermoiements néerlandais, il préféra envoyer son projet à Rome et à Bonn (É. DESCHAMPS, Belgium ..., op.cit., p.168). 
Toutefois, si la diplomatie belge n'a encore récolté que de maigres informations sur la position de la France, à l'exception du rejet poli du plan Spaak, un atout imprévu apparaît dans le jeu des agents belges: Olivier Wormser. Il s'agit du deuxième informateur notable, après Boegner. Suite à des entretiens franco-allemands en mai 1965, Wormser avait été favorable à une politique du moindre mal, selon laquelle la France concèderait, bon an mal an, une accentuation des pouvoirs de la Commission, en échange d'un plan de financement favorable à la France, concernant les questions agricoles.$^{52} \mathrm{Il}$ faisait donc partie de la frange des «pragmatiques». Cette dernière avait assisté au «revirement» de la France de juin 1965, pour des raisons d'ordre institutionnel, et sous l'impulsion du général de Gaulle. Ces dissensions franco-françaises avaient d'ailleurs semé la confusion la plus totale auprès des Cinq, en juillet 1965, mais aussi au sein même de la politique française.

Il s'avère que les déclarations de Wormser revêtent un intérêt particulier. En effet, il s'ouvre à Werner de Mérode et confie qu'une frange non-négligeable du Quai d'Orsay s'oppose à la ligne Couve de Murville-de Gaulle. Aucun diplomate français n'a été, selon lui, impliqué dans la note du 20 octobre, qu'il juge «très raide»; il ne voit aucune chance d'entente. L'Élysée a fait pression sur le Quai d'Orsay à la suite des indiscrétions relatives au plan Spaak dans Le Figaro et Le Monde, avant qu'il ne soit officiellement publié. De Gaulle ne souhaitait pas que ses agents fassent la moindre démarche vers Spaak: rien n'est envisageable avant les présidentielles. Après celles-ci, toujours selon Wormser, le général entamera des «initiatives tendant à extirper du système subsistant les derniers vestiges supranationaux, car [...], inquiet de son âge, [il] est pressé d'aboutir». ${ }^{53}$

Il convient toutefois d'accueillir les données fournies par Wormser avec un œil critique. En effet, il quitte prochainement ses fonctions à la DAEF. S'est-il senti ipso facto plus libre qu'à l'accoutumé, en allant jusqu'à contredire son ministre devant un diplomate étranger, en pleine crise? C'est fort probable. Notons que plus personne n'est dupe: la politique étrangère menée par de Gaulle s'aligne sur son agenda électoral. Si l'Élysée gagne du temps, le Quai d'Orsay - lui-même divisé en interne - mène plus que jamais les affaires à son propre train. Un train plus «spaakien» que celui de l'Élysée, ou, plus justement, moins «anti-spaakien». ${ }^{54}$

52. P.R. BAJON, Europapolitik 'am Abgrund'. Die Krise des 'leeren Stuhls' 1965-1966, Steiner, Stuttgart, 2012, pp.102-105; P.R. BAJON, De Gaulle finds his 'master'. Gerhard Schröder's 'Fairly Audacious Politics' in the European Crisis of 1965-66, in: Revue d'Histoire de l'Intégration Européenne, 2(2011), p.257.

53. P.R. BAJON, The Empty ..., op.cit., pp.214-218.

54. Toutefois, Jaspar semble oublier qu'à la fin 1964, l'image de Spaak en France s'était déjà sensiblement améliorée. Il s'exprime ainsi dans le bilan de 1965: «D'une année à l'autre, le changement de climat est notable. Naguère encore le ministre des Affaires Étrangères de Belgique passait pour l'adversaire le plus résolu de la politique élyséenne». AGR, FMHJ, n²533-2534, La France en 1965, pp.38-39. 


\section{L'Incident Bentinck: la fin de la parenthèse franco-belge}

À ce stade, la politique étrangère de la Belgique ne peut que faire un constat d'échec: aucune promesse de la France, échec du Plan Spaak et éventuel affaiblissement de Bruxelles au sein du Marché Commun, pour s'être trop rapprochée de la France durant la crise. C'est à ce moment qu'intervient l'incident Bentinck, qui, en somme, mettra fin au climat de confiance franco-belge de juillet-octobre 1965.

Tout commence par un article du Newsweek, paru le 25 octobre, faisant état de la position du général de Gaulle à propos de plusieurs problèmes internationaux. Il ne peut s'agir que d'une fuite..$^{55}$ Le journal américain, par le biais de son diplomatic correspondent, Edward Weintal, a obtenu «the following account of an hour-long talk that de Gaulle recently had with a distinguished diplomat who represents a country friendly to both the US and France»». ${ }^{56}$ L'article relate l'opposition du général à toute arme atomique en RFA, à toute influence de celle-ci dans la stratégie européenne et assure que la bombe française pourrait défendre Bonn. On y retrouve le refus du vote majoritaire, une possible alliance avec les Soviétiques et l'exigence du départ des soldats américains d'Europe. Le journaliste Roger Massip reprend et analyse l'article dans Le Figaro du 26 octobre. ${ }^{57}$ Le Quai d'Orsay s'empresse de nier ces déclarations et parle d'«affabulations grossières». 58

Qui est à l'origine de cette fuite? Jaspar mène son enquête et tient Davignon au courant; la fuite émanerait de l'ambassadeur des Pays-Bas à Paris, le baron Willem Carel van Bentinck, que Jaspar avait croisé le 24 septembre. ${ }^{59}$ Il note une remarque qui a dû déconcerter Davignon: «Le ton de l'article de l'hebdomadaire américain me frappe car il coïncide avec certains des propos que vous nous avez tenus samedi dernier»! ${ }^{60}$ Il est vrai que Bentinck a eu, le 18 octobre, un long entretien avec de Gaulle. ${ }^{61}$ Toutefois, le compte-rendu de leur conversation reprend la question du vote majoritaire, d'une révision des traités de Rome et de la vocation trop atlantiste des Pays-Bas. Mais, en revanche, il n'est fait aucune mention des troupes américaines ou

55. Déjà le 21 octobre, Couve de Murville avait confié à Wormser que la presse (française?) avait mésinterprété le contenu de l'entretien franco-néerlandais (J.-M. PALAYRET, De Gaulle ..., op.cit., p.64). Bien qu'A.G. HARRYVAN, A successful Defence of the Communautarian model? The Netherlands and the Empty Chair Crisis, in: J.-M. PALAYRET, H.S. WALLACE, P. WINAND (dir.), op.cit., pp.129-152 ne fasse aucune allusion à Bentinck, les archives néerlandaises devraient toutefois enrichir notre connaissance de ce personnage et de l'incident.

56. Newsweek, 25.10.1965.

57. Le Figaro, 26.10.1965.

58. La Dernière Heure, 26.10.1965.

59. AGR, FMHJ, n²637, Agenda 1965.

60. AGR, FMHJ, n²609, Jaspar à Davignon, 26.10.1965.

61. J.-M. PALAYRET, De Gaulle challenges the Community. France, the Empty Chair Crisis and the Luxembourg Compromise, in: J.-M. PALAYRET, H.S. WALLACE, P. WINAND (dir.), op.cit., p. 63 , fait allusion à cet entretien (qu'il date, par une coquille, du 8 octobre), mais ne fait aucune allusion à l'incident occasionné par le Newsweek. 
de la RFA. ${ }^{62}$ D'après Jaspar, suite à la visite d'un de ses conseillers à l'ambassade des Pays-Bas, Couve de Murville a rappelé Bentinck à de meilleures convenances.

Jaspar met dans la confidence André Molitor, chef de cabinet du roi Baudouin, et de Mérode (muté à la Direction Générale de la Politique). Il ignore si cette indiscrétion est orchestrée par La Haye. D'après ses sources, le télégramme a été envoyé par Bentinck aux milieux de la Communauté européenne à Bruxelles. La fuite viendrait de là. Il autorise de Mérode a diffuser l'information au département des Affaires étrangères, mais lui demande de taire ses sources. Le 27, la rédaction de L'Express contacte Jaspar. Elle lui demande à quand remonte son dernier entretien avec le général. L'hebdomadaire serait-il tenté de lui attribuer cette fuite par une sorte de phénomène épique? Jaspar a en effet déjà été mêlé à l'une ou l'autre affaire bénigne, relative à des indiscrétions commensales. Il dément; leur dernière rencontre remonte au 4 mai. ${ }^{63}$

Au-delà de l'anecdote, cet incident ne contribue pas à apaiser le climat francobelge.

\section{Une splendid isolation à la française?}

En parallèle, les débats vont bon train à l'Assemblée nationale française. Schumann propose de suspendre la règle du vote majoritaire, sans pour autant l'abolir. Habile dans ses termes et dans le choix de ses mots, Couve de Murville nie vouloir la révision des traités de 1957, alors qu'il ne s'agit que de cela. Jaspar sera curieusement moins sévère que Wormser à l'égard de cette prise de parole. Le 9 novembre, Wormser reparle d'une réunion des six ministres, qui se tiendrait à Bruxelles. Sans doute refroidi par l'incident Bentinck, le gouvernement français ne le souhaite pas, «à cause de l'atmosphère qui y règne et des habitudes d'indiscrétion que les fonctionnaires internationaux et les journalistes y ont contractées». Au contraire de Spaak, Couve de Murville souhaite commencer par des entretiens bilatéraux (et non multilatéraux), mais il promet la primeur à son homologue belge. Depuis le mois de juillet, les Belges bénéficient indubitablement d'une plus grande considération de la part de Paris que les Quatre autres. Cette réalité se confirme derechef. Mais, il n'empêche que nous sommes au cœur d'une crise européenne où les résultats probants de relance sont quasi nuls. Couve de Murville, effaçant au passage une de ses résolutions du 20 octobre, refuse toute discussion concernant le financement de la PAC avant que les Parlements des Six n'aient révisé les traités de $1957 .{ }^{64}$ Plus que jamais, Paris campe sur ses positions.

62. Entretien entre de Gaulle et Bentinck, 18.10.1965 in: Documents diplomatiques français, vol.28, t.II, Peter Lang, Paris, 2004, pp.460-462.

63. AGR, FMHJ, n²609, Jaspar à Davignon, 26.10.1965; Jaspar à Molitor et de Mérode, 26.10.1965; Jaspar à de Mérode, 27.10.1965.

64. AMAEB, n¹4642, Jaspar à Spaak, 27.10 et 09.11.1965. 
Le début de l'année 1966 est marqué par le Compromis de Luxembourg, mettant un terme à la crise ouverte le 30 juin 1965. Ce compromis réduit, d'une part, le rôle de la Commission, au bénéfice du Conseil des ministres et, d'autre part, la question de la majorité qualifiée n'est pas totalement évacuée. L'important, pour Spaak, était de sauver le Marché Commun. Une semaine après ce compromis, le 8 février, Boegner réoccupe son siège de représentant de la France auprès des Communautés. L'affaire se résorbe, certes provisoirement.

Jaspar confie ses impressions à l'ancien Premier ministre Paul Van Zeeland, à l'issue du compromis. À ses yeux, les réunions de Luxembourg n'ont pas mis un terme à l'isolement de la France. Ce dernier, jumelé à une distanciation progressive de Bonn, ne présage rien de bon pour l'ambassadeur. Permettons-nous de conclure par ce courrier, au ton quelque peu obsidional, mais dont la teneur est instructive:

«Les résultats, au Luxembourg, sont médiocres; en réalité, la France en est revenue, sous un aspect différent, au Plan Fouchet rejeté pour les raisons que tu connais. Je ne sais comment on en sortira mais l'isolement relatif de la France me fait craindre un isolement plus complet de l'Allemagne, dégagée des liens amicaux dans lesquels nous rêvions de l'enserrer. Récemment, un collègue de l'Est me décrivait comment l'emprise économique allemande se développe dans ces régions: cet ambassadeur disait que l'Allemagne de l'Ouest préparait un véritable plan Marshall pour les pays de l'Est. C'est la raison pour laquelle je pense que la France rêve de libérer les exportations de l'Est vers l'Hexagone. Je ne crois pas à l'OTAN, pas plus qu'aux chars, armes conventionnelles ou avions de papa, mais la présence des officiers d'État-major allemands à Fontainebleau me rassure, je redoute de leur donner involontairement une liberté dont ils profiteraient pour créer, dans une bonne petite ville de Bavière, un bon petit État-major allemand qui n'aura plus qu'une idée, das grossdeutschland (sic), quelle que soit l'étiquette, ou le drapeau». ${ }^{65}$

\section{Conclusions}

La politique étrangère de la Belgique à l'égard de la France lors de la crise de la chaise vide présente plusieurs caractéristiques. D'abord, elle est remarquable par sa volonté de réserver une certaine priorité à la poursuite de négociations entre Paris et Bruxelles, au niveau bilatéral. Toutefois, cette mise à l'écart du multilatéralisme divise sensiblement les agents diplomatiques belges; si l'ambassade à Paris prône, en août 1965, une démarche unilatérale, Étienne Davignon émettra certaines réticences à cette idée. Il apparaît que le choix d'opérer à un niveau bilatéral ait été privilégié par le fait que le Quai d'Orsay ait particulièrement apprécié les tentatives de Paul-Henri Spaak, en vue d'éviter la crise, dans la nuit du 30 juin au 1er juillet 1965. Face à la passivité italienne, l'opposition systématique des Néerlandais et la distanciation de Bonn, Paris semble accorder plus de crédit à Bruxelles. La diplomatie belge est dès lors consciente

65. CEHEC [Centre d'Étude de 1'Historie de 1'Europe Contemporaine], FVZ, n²095bis, Jaspar à Van Zeeland, 20.01.1966. 
qu'un rôle de médiateur lui est ipso facto offert. ${ }^{66}$ Puis, ce contact Paris-Bruxelles, bien que lourdement freiné par les «silences» de Maurice Couve de Murville (instigateur de cette chaise vide, avalisée par de Gaulle) et la postposition permanente d'un entretien franco-belge, a été favorisé par une image assez positive de Spaak telle qu'elle est véhiculée dans la presse française, même la plus gaulliste. Ce dernier point permet de nuancer le stéréotype du Spaak «mal-aimé» de la France. Si les années 1962-1963 consistent en un climax de l'antagonisme franco-belge sur l'Europe, retenons que les tentatives de relance de 1964-1965 furent presqu'unanimement bien accueillies par les périodiques de l'Hexagone. Cette tendance sera renforcée à la fin octobre 1965, lorsque Spaak apparaît presque comme un francophile, en contraste de Jean-Charles Snoy. Enfin, les Belges ont pu compter sur quelques informateurs français de première importance, alors que l'Élysée et le Quai d'Orsay s'arment de mutisme. Si le lien tissé entre Werner de Mérode et Jean-Marc Boegner aurait sans doute pu être exploité davantage, les impressions négatives d'Olivier Wormser sur la politique de son propre ministère montrent à quel point il existait une vive tension entre un nombre important de fonctionnaires du Quai d'Orsay et les autres acteurs de la politique étrangère française (Couve de Murville, leur supérieur, et de Gaulle). À cet égard, nous rejoignons certaines conclusions de P.R. Bajon.

Mais, la diplomatie belge a également été confrontée à certains obstacles qui, manifestement, ont empêché qu'elle puisse jouer pleinement ce rôle de médiateur et, in fine, convaincre la France de réoccuper, avant décembre 1965, la chaise vide de Boegner. Parmi ceux-ci, certains ressortent de la politique intérieure de chaque pays. Cette politique intérieure peut souvent avoir un impact significatif sur la politique étrangère. D'une part, côté belge, le contexte postélectoral, qui dura jusqu' au 27 juillet 1965, ne contribue pas à renforcer Spaak, dont le maroquin ministériel est hypothéqué. La période estivale ne représente pas non plus un élément positif en vue de faire avancer les affaires (absences de Couve de Murville, Jaspar, de Mérode). D'autre part, la politique intérieure française a pesé de tout son poids sur la crise et a induit les Belges en erreur. En effet, la diplomatie belge avait sans conteste sous-estimé à quel point le général de Gaulle avait aligné sa politique européenne sur le scrutin présidentiel de décembre 1965. Il s'agissait de la première élection au suffrage universel direct, en vertu de la révision constitutionnelle de 1962. Cet enjeu de poids, à l'Élysée, avait la primauté sur la question européenne. Or, Bruxelles a longtemps cru, jusqu'en septembre, qu'au contraire, cette échéance électorale donnerait aux Belges un moyen de pression sur Paris, que l'on forcerait à négocier sur l'Europe avant la période électorale. Encore en décembre 1965, Spaak avait espéré que la mise en ballotage de de Gaulle lors des élections l'amènerait à «plus de compréhension». ${ }^{67}$ Mais, dans l'esprit du général, ces élections avaient commencé en juin 1965. En gardant suffisamment longtemps secrète cette partie de son jeu, il a pu garder la main. Lorsque les Belges réaliseront la teneur de l'intime objectif du locataire du Faubourg Saint-Honoré, il sera déjà trop tard. En marge de ce facteur «politique intérieure», il

66. Spaak s'attribuera d'ailleurs le rôle de «conciliateur» entre Bonn, La Haye et Paris. P.H. SPAAK, Combats inachevés, vol.2: De l'espoir aux déceptions, Fayard, Paris, 1969, p.412.

67. Procès-verbal du Conseil des ministres de Belgique, 17.12.1965, p.531. 
en est des moins prévisibles, à l'instar de l'incident Bentinck d'octobre 1965, qui contribua à mettre un terme au dialogue franco-belge, jusqu'au Compromis de Luxembourg de janvier 1966.

La période suivant cette crise portera en elle plusieurs changements notables et fait office de charnière: départs de Paul-Henri Spaak, de Marcel-Henri Jaspar, d'Olivier Wormser, chute du gouvernement Harmel, crainte cyclique, chez certains diplomates belges, d'un regain nationaliste franco-allemand, et, surtout, en mars 1966, retrait français des états-majors intégrés de l'OTAN et transfert du SHAPE de la Porte Dauphine à Bruxelles. 